\title{
Noise Reduction on The Use of Different Ground Model on The EMI Filter of Reading LED Lighting for Passenger Train
}

\author{
Haryo Dwi Prananto*, Muhammad Imam Sudrajat, Priyo Wibowo, \\ Yoppy Yoppy, dan R. Harry Arjadi \\ Pusat Penelitian Teknologi Pengujian Lembaga Ilmu Pengetahuan Indonesia, \\ Tanggerang, Banten, Indonesia \\ *Corresponding author, e-mail: haryo.prananto@gmail.com
}

\begin{abstract}
Initial measurement showed that reading LED lighting used in this experiment produced excessive conducted emissions (CE). EMC filter is needed to suppress the CE noise. The LED light is AC powered through a two-wire cable without ground wire, whereas the EMC filter does have ground. Experiments were conducted to find out the attenuation effect of different filter's grounding connection methods. Four cases of grounding connections are measured and compared, i.e. unconnected ground, earth-connected ground, chassis-connected ground, and additional ground plate. Measurements showed that chassis-connected ground had the strongest attenuation effect. Also, for the additional ground plate configuration, it turned out that larger plates exhibited stronger attenuation compared to smaller plates.
\end{abstract}

Keywords : LED driver, EMC Filter, grounding, impedance, common mode

\section{Introduction}

In recent years utilization of LED lighting system in the passenger train is rapidly progressing. This system is very popular because it has higher energy efficiency compared with fluorescent lamp. On the other hand electromagnetic interference (EMI) from LED lighting system generate EMI noise and has potential to disturb other electronic devices inside the train. Hutomo et al [1] analyzed the radiated and Conducted Emission (CE) noise of 5 types LED lighting for passenger train application. Each type has different interference spectrum profiles characteristics. Nugroho et al [1] reveal that the 3watt LED reading lamp has highest CE noise, and it exceed CISPR 15 limits.

The CE noise from LED lighting mainly originate from its driver [2]. The LED driver contains a switching circuit that generates common mode and differential mode current [3]. Common mode and differential mode noise will propagate through conductor and become conducted emission noise to their environment or other interconnected system. To avoid this noise affecting the other systems, EMI from the source must be reduced and be attenuate.

There are many ways to reduce and attenuate noise in conducted emission; one method that already widely known is by adding a EMI filter. It effectively reduce differential mode noise and common mode noise using particular components and configuration. The particular components are common mode choke, differential mode choke, $\mathrm{X}$ capacitor for differential mode, and $\mathrm{Y}$ capacitor for common mode.

In general application an effective EMI filter has ground reference in its circuit. However, normally in a 3-watt reading LED lighting on the passenger train does not have real ground connection. Consequently, grounding method to reduce electromagnetic noise from this lighting will become tricky. In such cases, the EMI filter ground path cannot be connected to the LED driver. This condition usually is not enough to reduce EMI noise.

Chen et all [3] analyzed the effect of singlepoint grounding and series-connected grounding method to eliminate negative effect of common ground line. The result shows that parasitic inductance of common ground line can be reduced by series-connected grounding.

Araújo [4] characterizing conducted emission test for work LED light on application forestry wheeled harvesters with DC/DC buck convertion topology and in continuous current mode. He also used common mode power line shock nanocrysralline in his EMI Filter to reduce the emission accordance CISPR 25 Class 5. The result 
is that filter can reduce emission from LED in clas 5.

Wang et al [5] analyzed the parasitic effects of the grounding path on the EMI common mode filter on an electronic power system of a motor. They use theory of grounding and loop principle to analyze their experiment. Jeong et all [6] added a terminal ground filter between the cable ground and chassis in air conditioner application to reduce common mode noise in conducted emission. This method is effective to reduce conducted emission noise at low frequency.

EMC filtering is needed when LED light produces limit exceeding emissions. Some types of LED light used in passenger train are AC powered through a two-wire cable, which means it does not have ground wire. In such cases, it can be tricky to decide whether it is necessary to connect the filter's ground wire to achieve the required attenuation.

In compariosn with previous study, this study will propose the most effective grounding method that can be applied in lighting EMI filtering system that without unreal ground earth, like a reading LED lighting at passenger train. This study will discuss and analyze the attenuation effect of four cases of filter's grounding configurations: unconnected ground, earthconnected ground, chassis-connected ground, and additional ground plate.

\section{Theoretical Background}

\subsection{Ground Loop}

EMI coupling mechanism involving ground network is a looping mechanism. A ground loop can be causing two problems; first problem is noise voltage resulted from induced current that comes from magnetic coupling, and second problem is that the return current may take a path far away from the signal source. The ground loop can act as a receiving or transmitting antenna.

Ground loops give a high contribution to the propagation pattern of radio frequency (RF) energy. RF current naturally will attempt to find return path with smallest impedance value through any available medium or path and sometimes create undesired loop. This loop could cause a voltage difference between two devices [7]. In addition, existence of inductance in a transmission line produces interference coupling between source and victim [7].

The most important method to suppress EMI is optimizing ground as a $0 \mathrm{~V}$ reference plane and signal return loop. High-speed component, power line or RF generator components must be located as close as possible to ground structure to minimize currents loops and divert all unwanted signal to $0 \mathrm{~V}$ reference structure [7].

\subsection{Chassis Ground}

In some electronics equipment, conductor or metal which is connected to the metal enclosure called chassis ground [8]. Chassis ground can be a way to reduce electromagnetic interference and noise if it is connected in the right place. Consider the PCB circuit inside the metal enclosure and connected by input and output cable as shown in Figure 1. There is Voltage Drop $\left(\mathrm{V}_{\mathrm{G}}\right)$ in circuit ground caused by current and finite impedance incircuit ground. Common mode current from circuit ground is divided, and then it flows into cable and the PCB ground to chassis connection. The lower of chassis impedance, the lower the common mode current flow on to cable [8].



Figure 1. Scheme of chassis ground mechanism [8]

An ideal chassis ground should carry no current or will carry current only at fault condition. To minimize RF voltages and currents that generated between board and chassis, chassis ground must be connected as short as possible to the PCB ground plane [9]. This direct connection is important because RF signal can be suppressed by minimizing the flux area or magnetic loop that have approximately $1 / 20$ wavelength of the highest RF generated frequency [7].

\subsection{Common Mode and Differential Mode}

In electronic circuit common mode $(\mathrm{CM})$ and differential mode (DM) determine the amount of RF energy that is developed and propagated [7]. In the $\mathrm{CM}, \mathrm{RF}$ field presents on both signal and return current and propagates in the same direction. CM RF field is the foremost source of 
RF noise and harder to suppress. In the DM, RF field presents on both signal and return current and flow on the opposite direction. Illustration of CM current $\left(\mathrm{I}_{\mathrm{CM}}\right)$ and $\mathrm{DM}$ currents $\left(\mathrm{I}_{\mathrm{DM}}\right)$ propagation are shown in Figure 2 [7].
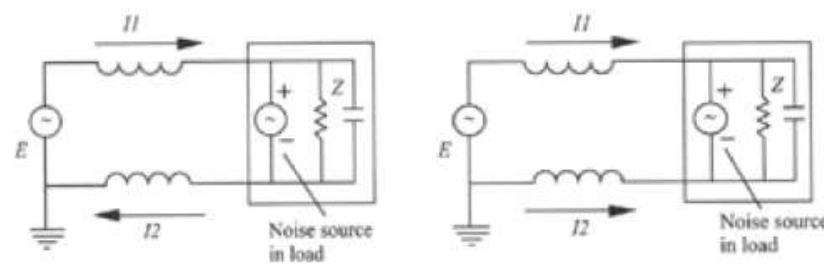

Figure 2. Differential mode (left) and common mode (right) current path [7]

In switched mode power supplies, including LED drivers, common mode noise is mainly generated by capacitive coupling mechanisms. The MOSFET is switching at high $\mathrm{dv} / \mathrm{dt}$, which is then capacitively coupled to heatsink or secondary side and eventually to ground. This common mode current finds its way back to the source (MOSFET) through both phase and neutral lines. In practical EMC, common mode current is measured by LISN: phase-to-ground and neutralto-ground. Therefore, in order to reduce the common mode emission, it is necessary to reduce the amount of capacitive coupling to ground. In other words, the common mode emission is to be confined and diverted back to the source as much as possible.

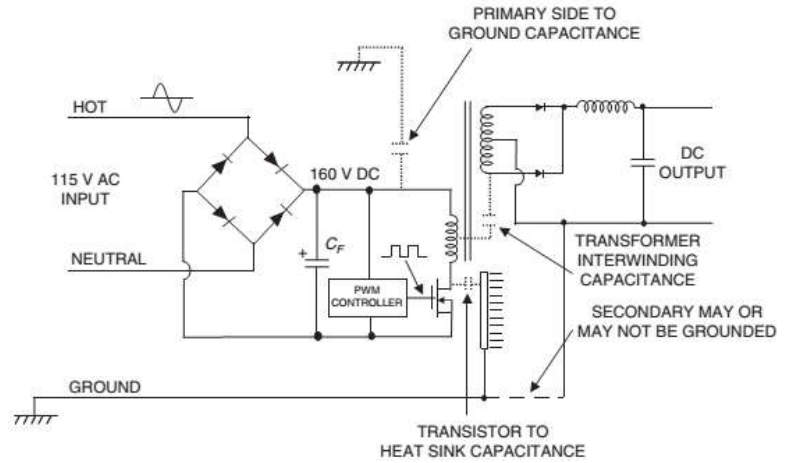

Figure 3. Common mode noise generation in switching power supply [9]

On the other hand, differential mode noise is generated by normal operating current flow. Generally in AC-DC switching power supplies, current flows from phase line to diode bridge and charges input capacitor. Then current from capacitor is flowing through inductor, MOSFET, and back to capacitor. The current is flowing in a switching manner at frequency of $20 \mathrm{kHz}-1 \mathrm{MHz}$, and thus results in high frequency ripple noise, whose level is depending on the parasitic inductance and resitance of the input capacitor.

\subsection{LED Drivers}

LED drivers are a stand-alone control circuitry that provides and regulates power to make LED operate properly in consistent performance [9]. Constant current driver and constant voltage driver are both feasible options for LED driver, the dissimilarity between them is the method in which they deliver the power.

Constant voltage LED drivers are designed for a single output voltage but cannot provide current limiter. The LED configuration for Constant Voltage LEDs is always wired parallel. When applied to two lamps or more, the current drawn from the driver is rise up in accordance with the number of lamps.

Constant current LED drivers are intended for operating in fixed current and vary of voltage output in designated range. This driver eliminates variations in current due to changes in forward current [9]. Constant current driver required to supply current is usually in milliamps (mA) or amps (A). The higher current level will make the LED brighter, but if not kept constant, the LED will draw more current than its maximum capability which results in reducing LED life spans or damage the LED due to increased temperature. Constant current driver is a common way to drive high power LEDs and maintains stable brightness of series LED.

\section{Method}

The research method is an experimental method which is conducted in EMC laboratory. The LED lights used in this research is a reading LED light on passenger train.

The LED driver is connected to Wurth Electronics general multiple line EMI filter [10]. Balanced $\pi$ filter type is used and the circuit can be seen in Figure 6.

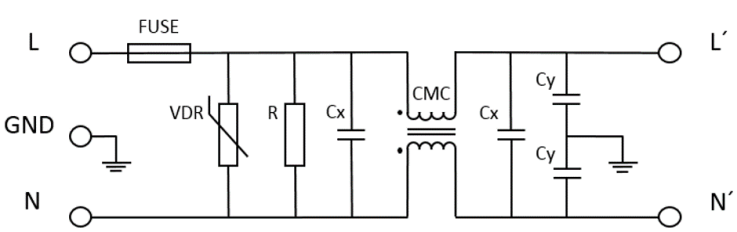

Figure 6. Multiple Line Filter [10] 
This filter used LC passive componenct which may can reduce current harmonic in LED [11]. Cx and $\mathrm{Cy}$ values are $0.47 \mu \mathrm{F}$ and $2.2 \mathrm{nF}$, respectively. The common mode inductor value is $47 \mathrm{mH}$ and resistor value is $1 \mathrm{M} \Omega$. Cx capacitor is used to attenuate noise from differential mode and $\mathrm{Cy}$ is used to attenuate common mode noise.

The noise level in conducted emission test is measured by spectrum analyzer in the frequency range of $9 \mathrm{kHz}-30 \mathrm{MHz}$ referring to CISPR15:2013 standard. The object of this measurement is the reading LED lighting with and without EMI filter.

As mentioned previously, the driver's AC input actually does not come with ground wire, whereas the EMC filter has one. This leads to questions about the filter effectiveness on connecting or disconnecting its ground wire. In this paper, four cases of grounding are put on experiments.

In case I, the EMC filter's ground is left unconnected, as shown in Figure 4. In case II, the filter's ground is connected to the earth ground, to which LISN ground also connects. The driver AC input is fed by phase dan neutral lines, like shown in Figure 5. In case III, as depicted in Figure 6, the filter's ground is connected to a metal plate, which is placed $5 \mathrm{~cm}$ apart from the LED light. The plate is not referenced to earth ground. In case IV, which is shown in Figure 7, the filter's ground is connected to the LED light chassis. The metal chassis is coupled back-to-back with the driver, but is not electrically connected to each other.

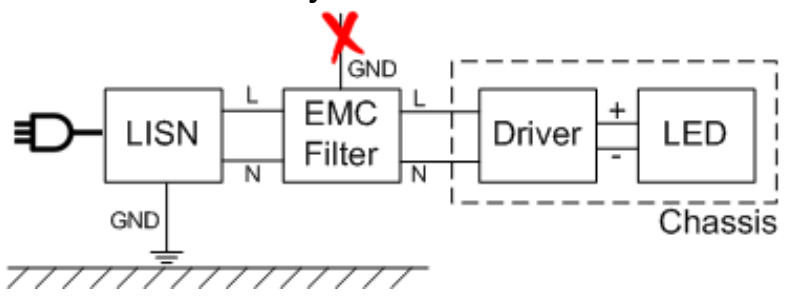

Figure 4. Case I: EMC filter's ground is left disconnected

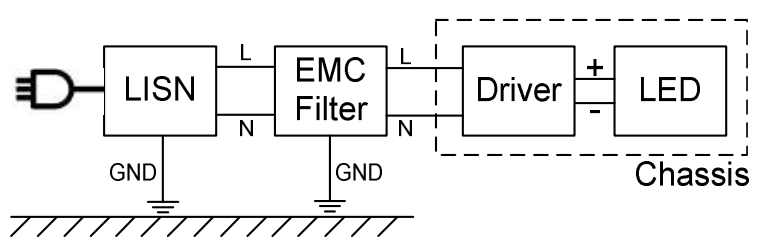

Figure 5. Case II: filter's ground is connected to earth ground

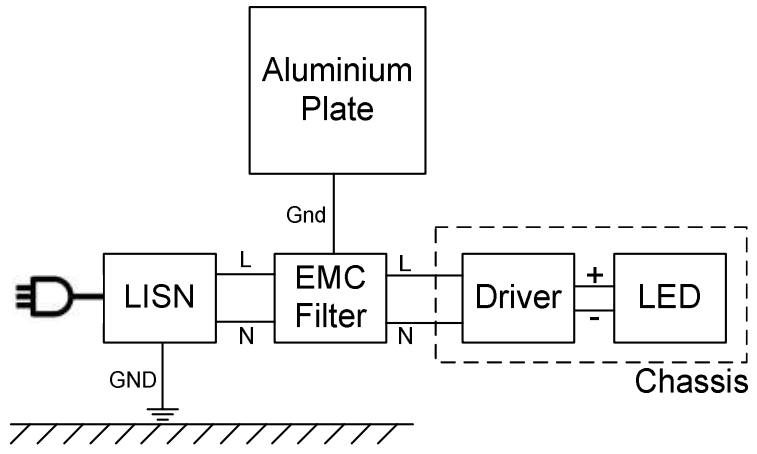

Figure 6. Case III: filter's ground is connected to a metal plate

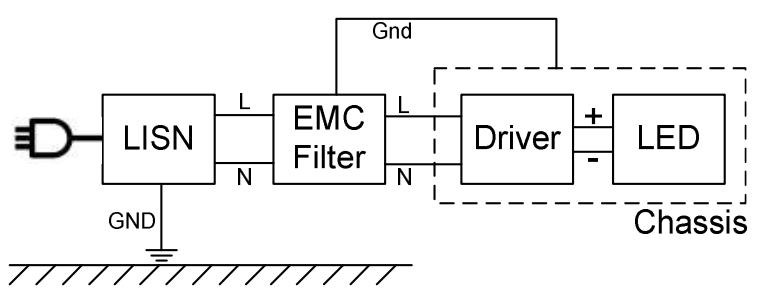

Figure 7. Case IV: filter's ground is connected to LED lighting's chassis

Particularly in case III, conducted emission tests are measured with three different plate area i.e. $20 \times 20 \mathrm{~cm}^{2}, 40 \times 40 \mathrm{~cm}^{2}$, and $60 \times 60 \mathrm{~cm}^{2}$. The position and distance between EMI filter and the aluminium plate are maintained constant.

\section{Result and Discussion}

\subsection{Comparison of conducted emission test with and without EMI filter}

Figure 8 shows a comparison of conducted emission spectrum from reading LED light with filter (according to case I) and without EMI filter. Conducted emission noise is caused by common mode current and/or differential mode current.

As shown by the blue trace in Figure 8, the EMI filter is effective to attenuate noise in frequency range of $0.1-30 \mathrm{MHz}$. Although, at frequency of around $2-9 \mathrm{MHz}$ and at frequency $28 \mathrm{MHz}$ the noise still appears and potentially exceeds the limit of standard. High frequency emission is usually caused by common mode noise. As in case I, because the filter's ground is left disconnected, the common mode capacitor $\mathrm{Cy}$ is in effect not used, and thus the common mode noise is only suppressed by the common mode choke. This results in less attenuation effect to the common mode noise. 
On the other hand, it can be seen that low frequency emission, which is usually dominated by differential mode noise, starting from the fundamental switching frequency of $30 \mathrm{kHz}$ to several higher order harmonics, is effectively suppressed. The attenuation is performed by the capacitor $\mathrm{Cx}$ which shunts or diverts the differential mode switching noise back to the source. In effect, LISN sees low differential mode voltage on the power lines.

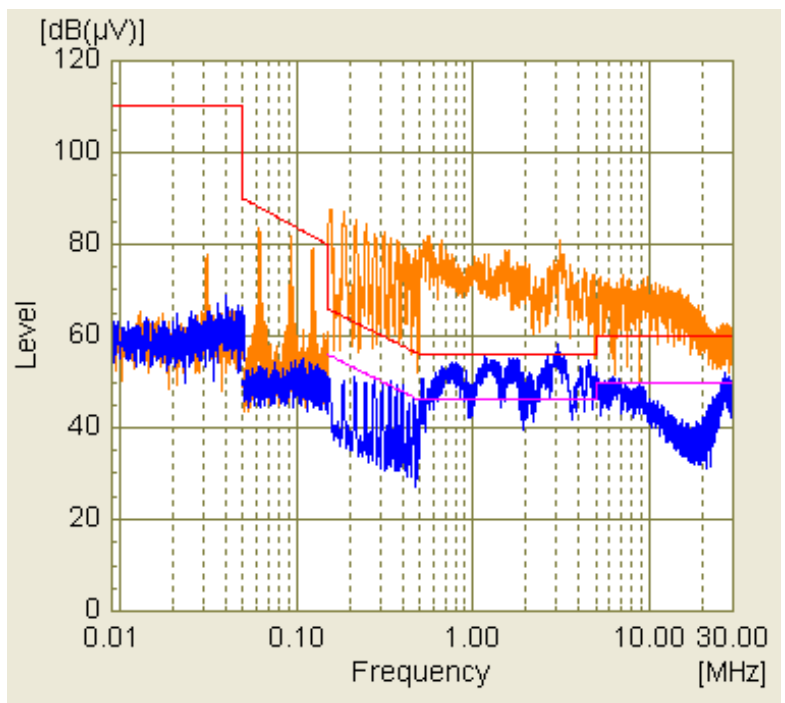

Figure 8. Conducted emission of reading LED light with (blue) and without (orange) EMI filter

\subsection{Comparison of conducted emission test in case III with different surface area}

Figure 9 shows spectrum profiles of three different plate areas. The green, orange, and blue traces are emission from reading LED according to case III setup with a plate area of $20 \times 20 \mathrm{~cm}^{2}$, $40 \times 40 \mathrm{~cm}^{2}$, and $60 \times 60 \mathrm{~cm}^{2}$, respectively. Overall, the $60 \times 60 \mathrm{~cm}^{2}$ plate shows the lowest emissions compared to other plate sizes. EMI noise attenuation becomes more effective when the plate is larger. This can be explained by the fact that larger plates facilitates stronger capacitive coupling from the noise source (LED driver) to the filter's ground. In effect, coupling to the measurement site's ground plane, and eventually to the measuring LISN is reduced.

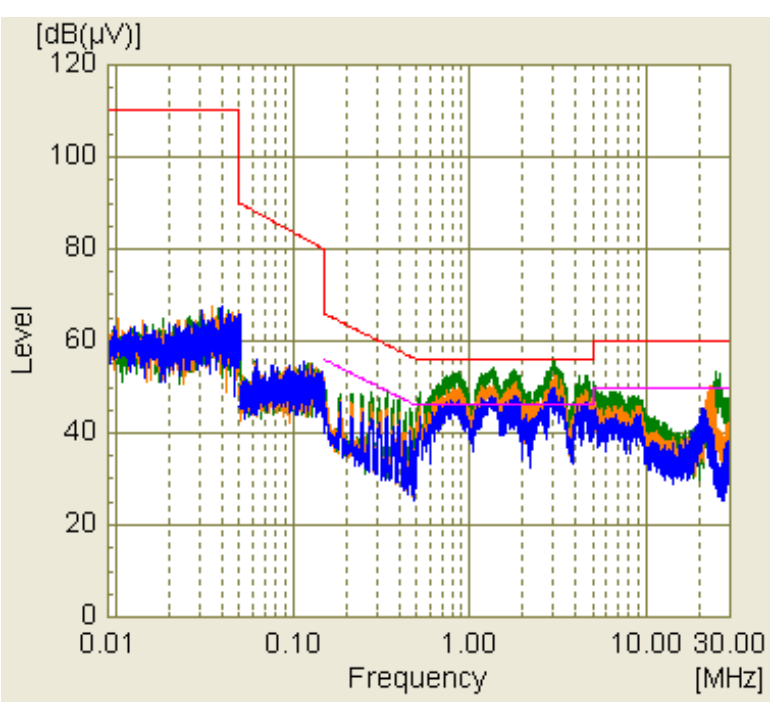

Figure 9. Result of CE test with different plate area: $20 \times 20 \mathrm{~cm}^{2}$ (green), $40 \times 40 \mathrm{~cm}^{2}$ (orange), $60 \times 60 \mathrm{~cm}^{2}$ (blue)

The result can also be explained by the concept of coupling path impedance. Current will always flow into the path which has least impedance. It is known that capacitance is proportional to the plate area. Larger plate area means higher capacitance, which then provides lower path impedance for capacitive coupling to take place.

Common mode noise generated in the LED driver has two routes to find its way back to the source. It can capacitively flow through the measurement site's ground plane and also through the metal plate, which is placed side by side with the driver. Therefore, more current will flow into larger plate, and less current flows into the measurement site's ground plane. As a result, lower voltage is measured in the LISN.

In addition, another difference can be seen in the $\mathrm{CE}$ spectrum in Figure 9 is peak frequency shifting around $20 \mathrm{MHz}-30 \mathrm{MHz}$. The graph shows that larger plate area tends to shifts down the peak frequency. Table 1 shows the frequency shift from the peak of each plate area. This may be related to LC resonance frequency. Larger plates have larger capacitances, which then have lower resonance frequency.

Tabel 1. Suspected List of Conducted emission Test of EMI filter with Variation Area of Aluminium Plate

\begin{tabular}{|c|c|c|}
\hline $\begin{array}{c}\text { Dimension of } \\
\text { Aluminium Plate }\left(\mathrm{cm}^{2}\right)\end{array}$ & $\begin{array}{c}\text { Freq. } \\
(\mathrm{MHz})\end{array}$ & $\begin{array}{c}\text { Level } \\
(\mathrm{dBuV})\end{array}$ \\
\hline $20 \times 20$ & 24.4949 & 53.3 \\
\hline $40 \times 40$ & 23.1815 & 50.1 \\
\hline $60 \times 60$ & 20.4949 & 45.5 \\
\hline
\end{tabular}




\subsection{Comparison of conducted emission test in case II, III, and IV}

Figure 10 shows a comparison of conducted emission test result from reading LED lighting according to setup in case II, III, and IV.

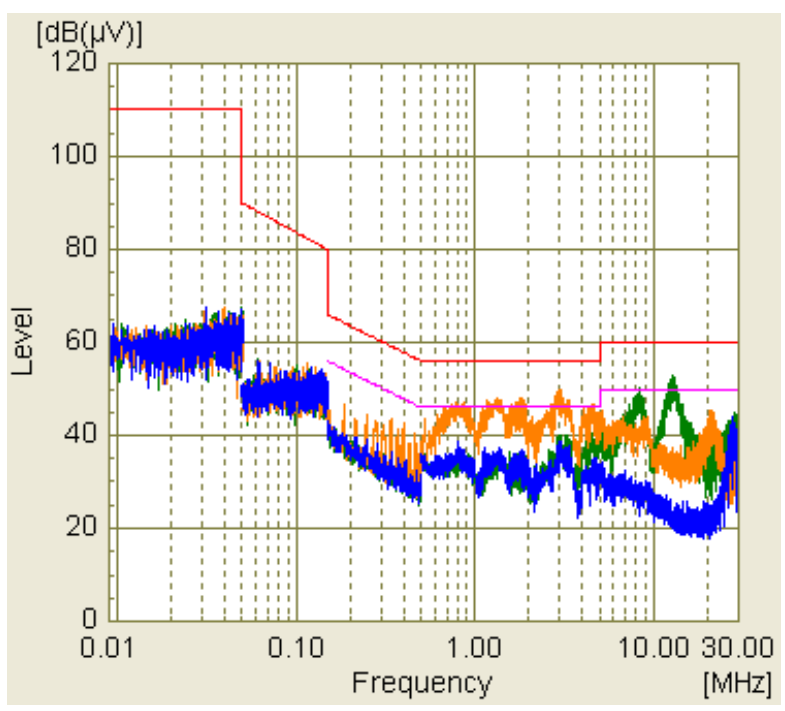

Figure 10. Result of CE test from reading LED in case II (green), case III - 60x60 $\mathrm{cm}^{2}$ (orange), and case IV (blue)

The green trace in Figure 10 is CE result from the case II. Meanwhile, the orange trace shows the result of conducted emission of case III with $60 \times 60 \mathrm{~cm}^{2}$ metal plate. Whereas the blue trace is the result from case IV where the filter's ground is connected to the LED light chassis.

The result shows that the LED light with EMI filter's ground connected to chassis has the lowest emission from other ground configurations. This happens because common mode noise generated from the LED driver is tightly coupled to the chassis. Then, the noise finds the least impedance through conductive couipling to the filter's ground. In the frequency range of $\mathrm{CE}$ measurements, which is upto $30 \mathrm{MHz}$, conductive coupling provides much lower impedance compared to capacitive coupling. Therefore, capacitively coupled noise to the LISN is minimal.

In both case II and case III, there is no conductive coupling between common mode noise source and the filter's ground. In case II, common mode noise has two ways to return to the source, i.e. to filter's ground and to LISN's ground. Both are through capacitive coupling. In case III, common mode noise return path is through capacitive coupling to the metal plate and
LISN's ground. Therefore, attenuation effects in both cases are sub-optimal.

It is worth to mention that in the experiment of case IV the filter's ground is not connected to earth ground. For safety reasons in practical usage, the filter's ground is usually connected to earth ground too, in addition to connected to the LED light chassis.

\subsection{Comparison of conducted emission test of reading LED lighting without filter and with filter in case $I$ and case IV}

Figure 11 again summarizes the effectiveness of filter in suppressing conducted emission. The figure also shows the difference between disconnected filter's ground (case I) and connected to the LED light chassis one (case IV). It can be seen that when EMI filter is used but its ground path is not connected (case I), the conducted EMI noise is decreased but the attenuation is not optimal. But when the filter's ground is connected to chassis the attenuation improves significantly.



Figure 11. Result of CE test from reading LED light without EMI filter (orange) and with filter in case I (green) and case IV (blue)

\section{Conclusion}

Additional EMI filter is needed to reduce conducted EMI noise from reading LED lighting at train passenger application. It has been shown that grounding technique of EMI filter also plays an important role in maximizing the filter attenuatuion effects. 
This research demonstrates that the effectiveness of EMI filter in suppressing conducted EMI from reading LED lighting for passenger train highly depends on the filter's grounding method. Comparing four grounding connection models, chassis-connected ground is the most effective one to attenuate conducted EMI noise. Appropriate grounding connection would result in highest attenuation.

\section{Acknowledgement}

The authors would like to thank to the Ministry of Research Technology and Higher Educations for sponsoring INSINAS No. 088/P/RPLLIPI/INSINAS-1/III/2018 research funding.

\section{Reference}

[1] H. W. Nugroho, P. Wibowo, R. H. Arjadi, H. D. Prananto, and M. I. Sudrajat, "Characteristics of Electromagnetic Interference LED Lightings for Passenger Train," in 2017 International Multidisciplinary Conferences on Productivity and Sustainability, Jakarta, 2017, pp. 43-46.

[2] P. Wibowo, H. D. Prananto, Y. Yudhistira, Y. Yoppy, M. K. Anam, and R. H. Arjadi, "Characterization of Electromagnetic Compatibility Performance of Trains LED Lighting by Mapping of Radiated Interference Distribution," in 2017 International Multidisciplinary Conferences on Productivity and Sustainability, Jakarta, 2017, pp. 39-42.

[3] H. Chen, P. Meng, and J. Li, "SeriesConnected Grounding of Common-Mode EMI Filter," IEEE Trans. Electromagn. Compat., vol. 52, no. 4, pp. 1066-1068, 2010.

[4] C. M. Araújo, "Characterization of electromagnetic interference conducted in DC-DC Buck Converter LED Light in accordance with CISPR 25 - Class," in 2018 13th IEEE International Conference on Industry Applications, Sao Paulo, Brazil, 2018, pp. 1259-1265.

[5] S. Wang, S. Member, Y. Y. Maillet, and F. Wang, "Parasitic Effects of Grounding Paths on Common-Mode EMI Filter's Performance in Power Electronics Systems," IEEE Trans. Ind. Electron., vol. 57, no. 9, pp. 3050-3059, 2010.

[6] S. Jeong, J. Kim, Y. Bek, and G. Park, “A Terminal Ground Filter between Cable and Chassis for Reduction of Conducted
Emissions at a Home Appliance," 2018 IEEE Symp. Electromagn. Compat. Signal Integr. Power Integr. EMC SI PI, pp. 414-418, 2018.

[7] M. I. Montrose, Printed Circuit Board Design Techniques for EMC Compliance - A Handbook for Designers, Second Edition, Second. New York: John Wiley \& Sons, Inc, 2000.

[8] H. W. OTT, Electromagnetic Compatibility Engineering. New Jersey: John Wiley \& Sons, Inc., 2009.

[9] G. Held, Introduction to Light Emitting Diode Technology and Aplications. Boca Raton, 2009.

[10] "Multiple Line Filter," Wurth Electronik, 2018. [Online]. Available: https://www.weonline.com/web/en/electronic_components/pr odukte_pb/demoboards/designyouremcfilter/li nefilter_744998_1 1.php?blank=.

[11]M. R. A. Cahyono, "Analisa Pemanfaatan Harmonisa Sebagai Sumber Energi Alternatif dengan Filter Pasif," J. Nas. Tek. Elektro, vol. 7, no. 3, pp. 161-168, Nov. 2018. 